\title{
Anti-inflammatory effects of non-statin low-density lipoprotein cholesterol-lowering drugs: an unused potential?
}

\author{
Anders Hovland ${ }^{a, b}$, Kjetil Retterstøl ${ }^{c, d}$, Tom Eirik Mollnes ${ }^{b, e, f, g, h} \quad$ Bente Halvorsen ${ }^{g, i}$, \\ Pål Aukrust ${ }^{g, i, j}$ and Knut Tore Lappegård ${ }^{a, b}$
}

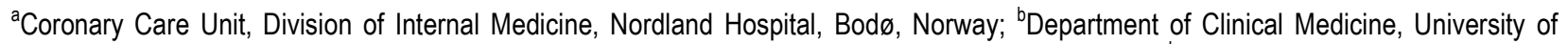

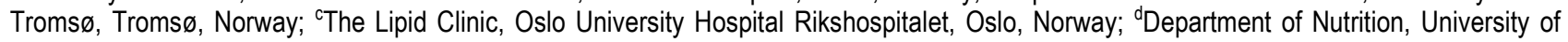
Oslo, Oslo, Norway; ${ }^{e}$ Centre of Molecular Inflammation Research, Norwegian University of Science and Technology, Trondheim, Norway; ${ }^{f}$ Research Laboratory, Nordland Hospital, Bodø, Norway; IInstitute of Clinical Medicine, Faculty of Medicine, University of Oslo, Oslo, Norway; ' Institute of Immunology, Oslo University Hospital Rikshospitalet, University of Oslo, Oslo, Norway; i'Research Institute of Internal

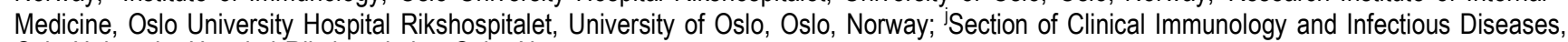
Oslo University Hospital Rikshospitalet, Oslo, Norway

\begin{abstract}
Objectives. Inflammatory responses are closely knit with low-density lipoprotein (LDL)-cholesterol in driving atherosclerosis. Even if LDL-cholesterol is causative to atherosclerotic diseases and LDLcholesterol lowering reduces hard clinical endpoints, there is a residual risk for clinical events, possibly driven by inflammatory processes, in accordance with its role in autoimmune diseases. Design. As LDLcholesterol treatment targets are reduced, the use of non-statin lipid-lowering drugs will probably increase. Atherosclerotic plaques evolve through lipid infiltration and modification in the intima, furthermore infiltration of cells including monocytes, macrophages, T-lymphocytes and neutrophils initiating inflammatory signaling. Here we briefly review inflammation in atherosclerosis and the effects of the non-statin lipid-lowering drugs on inflammation. The review is limited to the most common nonstatin lipid lowering drugs, i.e. proprotein convertase subtilisin-kexin type 9 (PCSK9) inhibitors, bile acid sequestrants (BAS) and cholesterol absorption inhibitors. Results. PCSK9 inhibition is mostly studied together with statins and is associated with a reduction of pro-inflammatory cytokines. Furthermore, PCSK9 inhibitors seem to have an effect on monocyte migration trough CCR2. They also have an interaction with sirtuins, possibly offering a therapeutic target. BAS have several interesting effects on inflammation, including reduction of pro-inflammatory cytokines and a reduction of the number of infiltrating macrophages, however there are relatively few reports considering that these drugs have been on the market for decades. Ezetimibe also has effects on inflammation including reduction of pro-inflammatory cytokines and adhesion molecules, however these effects are usually accomplished in tandem with statins. Conclusion. This topic adds an interesting piece to the puzzle of atherosclerosis, indicating that PCSK9 inhibition, BAS and ezetimibe all affect thromboinflammation.
\end{abstract}

\section{Introduction}

Inflammatory responses are closely knit with low-density lipoprotein (LDL) -cholesterol in driving the atherosclerotic process [1]. Both innate and adaptive inflammatory responses are important in evolving atherosclerosis through a bidirectional interaction with lipids [2,3]. Recently a close cross-talk between hemostasis and inflammation has been described, named thromboinflammation, with a main potential for further research in defining targets for inhibiting this cross-talk $[4,5]$.

There seems to be a residual risk for clinical events in a large proportion of patients with atherosclerotic diseases, possibly in part driven by inflammatory processes [6,7]. This hypothesis has recently been tested, and reduction of the pro-inflammatory interleukin (IL)-1b pathway by canakinumab reduced clinical endpoints $[8,9]$. On the other hand, in a recent randomized controlled trial, low dose methotrexate did not reduce risk of cardiovascular disease, [10] illustrating the importance of targeting the driving inflammatory pathways when using an anti-inflammatory approach in atherosclerotic patients.

Several reviews have discussed the anti-inflammatory effects of statins [11-14], a subject which is beyond the scope of the present review. However, the recent discovery of the importance of the IL-1b pathway in atherosclerosis [8] raises new questions about how other lipid-lowering drugs affect inflammation. In the present article we briefly review the effects of the non-statin lipid-lowering drugs on the inflammatory arm of atherosclerosis. The review is limited to the most common non-statin lipid lowering drugs: Proprotein convertase subtilisin-kexin type 9 (PCSK9) inhibitors, bile acid sequestrants (BAS) and cholesterol absorption inhibitors. 


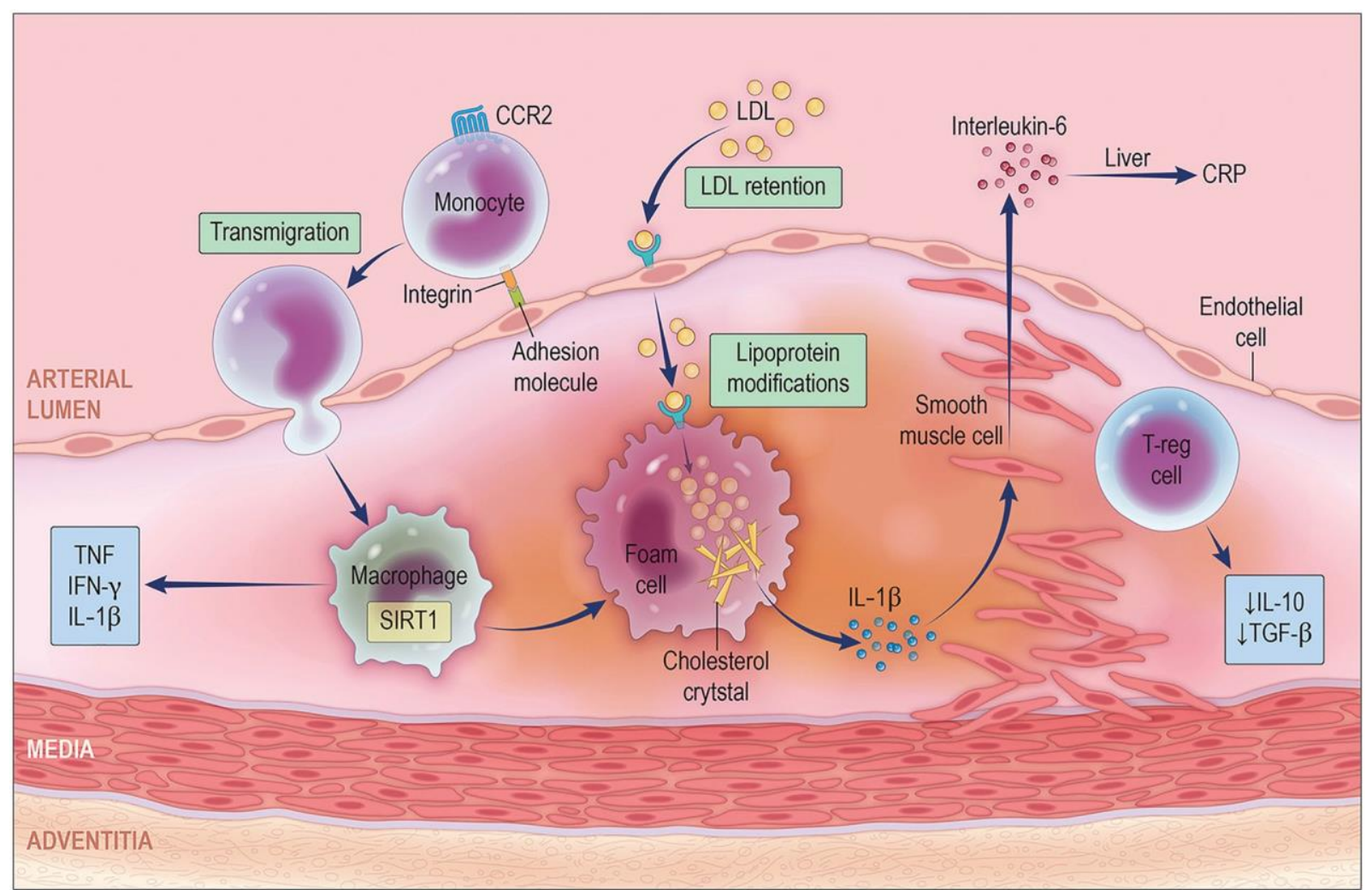

Figure 1. Monocytes enter the subendothelial space, and transform to different subsets of macrophages. LDL-cholesterol particles enter the intima and undergo modifications. Ultimately, lipid rich foam cells are formed being part of the atherosclerotic plaque. Several immunological responses are essential parts of the build-up of an atherosclerotic plaque. CCR2: C-C chemokine receptor type 2; LDL: low-density lipoprotein; CRP: C-reactive protein; TNF: Tumor necrosis factor; IFN: Interferon; IL: Interleukine; SIRT: Sirtuin; TGF: Transforming Growth Factor.

\section{Inflammation and the immune system in atherosclerosis (Figure 1)}

Already back in the mid nineteenth century, Virchow described the inflammatory nature of atherosclerotic plaques [15]. However, it was not until the late twentieth century that this research really picked up speed [16]. Inflammation is a major component of atherosclerosis [3,17], and cholesterol and inflammation have been described as partners in crime during atherogenesis [18]. In fact, like many other non-infectious disorders, atherosclerosis is characterized by a low-grade non-resolving inflammation with the interaction between inflammation and lipids as its hallmark.

In brief, lipoproteins are retained by matrix proteoglycans in the intimal layer of the arterial wall. These lipoproteins are prone to undergo oxidative modifications [19], and this event is followed by an immediate innate immune response [20]. Circulating monocytes migrate to atherosclerotic plaques, aided by a wide range of adhesion molecules and chemokine receptors [21]. Macrophages, prototypical cells in the innate immune system, have for several years been known to play a key role in lipid accumulation and inflammation during atherogenesis and more recent studies have delineated several macrophage subtypes within the lesion, such as pro-inflammatory M1 macrophages and the anti-inflammatory and pro-resolving M2 macrophages. This macrophage diversity has hampered targeted therapy directed against these cells in atherosclerotic disorders [22]. Moreover, we and others have shown that the NLRP3 inflammasome in macrophages play a major role in translating lipid-mediated (i.e. cholesterol crystals) signalling into inflammation with IL-1b and IL-18 as the major inflammatory outcome, and recent studies suggest that also complements are involved in these inflammatory responses [23]. Several other types of immune cells are also involved in atherosclerosis, including $\mathrm{T}$ and $\mathrm{B}$ cells $[24,25]$. Thus, whereas Th1 CD4p T cells and CD8p T cells seem to enhance atherogenesis, Th2 cells and in particular regulatory $\mathrm{T}$ cells seem to have anti-atherogenic properties at least partly related to the release of anti-inflammatory cytokines like IL10 and for regulatory $\mathrm{T}$ cells, also transforming growth factor. A similar diversity seems also to exist within the B cells [26].

Furthermore, neutrophils are pivotal in inflammatory responses in atherosclerosis [27] including in the formation of neutrophil extracellular traps [28], and these cells seem to be of particular importance in relation to plaque erosion, an important mechanism for the development of non ST elevated myocardial infarction (NSTEMI). Also mutations of myeloid cells in the bone marrow may give rise to clones of myeloid cells in peripheral blood called clonal haematopoiesis of indeterminate potential increasing the risk atherosclerosis possibly by regulating expression of inflammatory genes [1,29]. Interestingly, the genes that are most often mutated in these patients (e.g. DNMT3A and TET2) are related to regulation of epigenetic modification, suggesting an important role for methylation and demethylation of genes in atherogenesis $[30,31]$. 
This complex interaction between lipids and inflammation include activation of a variety of both inflammatory and anti-inflammatory pathways, and how to modulate this complex network of interacting molecules in an anti-atherogenic direction is a major task in the management of patients with atherosclerotic disorders.

Even though LDL-cholesterol lowering therapy has proven its excellent value in several clinical trials with significant reductions in major clinical endpoints, patients with atherosclerotic diseases treated with such medication still have a residual increased risk for new events, and this is particular true in patients with residual untreated inflammation. Whereas specific anti-inflammatory therapy such as IL-1 directed therapy may be needed, LDL-lowering therapy may also modulate the inflammatory arm of atherosclerosis both indirectly by attenuated the LDL-cholesterol mediated inflammation, but potentially also by directly modulating inflammatory signalling. Whereas these mechanisms are widely studied in statins, the possible anti-inflammatory effects of other lipid lowering medication are less known.

\section{Mechanisms of action, effect on LDL-cholesterol and clinical endpoints for commonly used lipid-lowering drugs beyond statins (Table 1)}

\section{PCSK9 inhibitors}

The use of PCSK9 inhibitors increases steeply. Currently there are two monoclonal antibodies in clinical use that inhibit PCSK9; evolocumab and alirocumab. They lower LDL-cholesterol by $50-60 \%$ though inhibition of PCSK9 and hence increases LDL-receptor capacity [32,33]. This leads to a reduction in cardiovascular clinical endpoints [34,35]. In the recent European Guidelines on Dyslipidemias, PCSK9 inhibitors are recommended as the third lipid lowering agent if the treatment targets are not met by statins and ezetimibe [36].

\section{Bile acid sequestrants (BAS)}

The most commonly used BAS in LDL-cholesterol lowering are colestipol, colsevelam and cholestyramine. Even if sevelamer is a BAS and also lowers LDL-cholesterol, it is most commonly used as a phosphate binding drug in renal failure. BAS have been in clinical use for decades. They lower LDL-cholesterol by stimulating bile synthesis and lowering intestinal lipid absorption, and they also affect glucose homeostasis in type 2 diabetes [37]. LDL-cholesterol is lowered by $15-20 \%$, and there is one primary prevention study showing effect on clinical endpoints [38]. However, endpoint studies have not been performed after the introduction of statins.

\section{Cholesterol absorption inhibitors}

Ezetimibe is the only clinically available cholesterol absorption inhibitor, however phytosterols also inhibit cholesterol absorption. Ezetimibe inhibits the Niemann-Pick C1-like protein in the small intestine [39], and the LDL-cholesterol lowering effect is $20-25 \%$ (fixed dose). In the IMPROVE-IT trial, clinical endpoints were reduced when adding ezetimibe to statins after acute coronary syndromes [40], and similarly in the EWTOPIA 75 primary prevention trial, ezetimibe reduced composite endpoints [41].

\section{Effects on inflammation for commonly used lipid- lowering drugs}

\section{PCSK9 inhibitors}

PCSK9 inhibitors as monotherapy or added to statins and ezetimibe efficiently reduce LDL-cholesterol, although CRP remains unchanged [42]. However, this does not exclude anti-inflammatory effects of PCSK9 inhibition that are not mirrored by CRP reduction.

Tang et al. interestingly demonstrated that oxidized LDLcholesterol upregulated PCSK9 in macrophages as well as inflammatory markers including IL-1a, IL-6 and TNF. These markers were reduced when inhibiting PCSK9 with small interfering RNA PCSK9 through nuclear factor kappalight-chain-enhancer of activated B cells [43]. The same group has also shown that PCSK9 is upregulated in murine atherosclerotic plaques, whereas downregulation leads to decreased plaques and decreased inflammation as measured by reduced concentrations of IL-1a, IL- 6 and TNF [44].

Bernelot Moens et al. found that persons with familial hypercholesterolemia with grossly elevated LDL-cholesterol had a three-fold increase in expression of the pro-atherogenic chemokine receptor CCR2 compared to controls with normal LDL-cholesterol [45]. When these subjects were treated with PCSK9 inhibition, the levels of LDL were reduced as predicted, and notably, CCR2 expression and TNF production was reduced, while IL-10, commonly considered an anti-inflammatory cytokine, was increased [45]. This finding of reduced CCR2 expression with PCSK9 inhibition might indicate the possibility of reduced monocyte influx to atherosclerotic plaques [21]. In the cell model of vascular smooth muscle cells, Grune et al. have demonstrated that PCSK9 levels are linked to LDL-cholesterol receptor mediating CCR2 expression [46], again pointing to a possible effect of PCSK9 inhibition on monocyte influx.

Sirtuins are a highly conserved protein family of histone deacetylases that are of major importance for mediating the beneficial effects of calorie restriction on cardiovascular disease, and seem to be key enzymes in regulation of metabolic driven inflammation [47]. These effects are partly related to

Table 1. Mechanism of action and LDL-cholesterol lowering effect of lipid lowering treatment.

\begin{tabular}{llcc}
\hline & \multicolumn{1}{c}{ Mechanism of action } & Effect & Endpoint studies \\
\hline PCSK9 Inh & Increases LDL-R activity & Up to 50-60\% & Yes [33,34] \\
BAS & Increase hepatic LDL-C clearance & Up to 20\% & Yes [38] \\
Ezetimibe & Inhibits intestinal LDL-C absorption & Up to 20-25\% & Yes [30] \\
\hline PCSK9 Inh: Proprotein convertase subtilisin-kexin type 9 inhibitors. & & \\
BAS: Bile acid sequestrant; LDL-C: low-density lipoprotein-cholesterol; LDL-R: low-density lipoprotein-receptor.
\end{tabular}




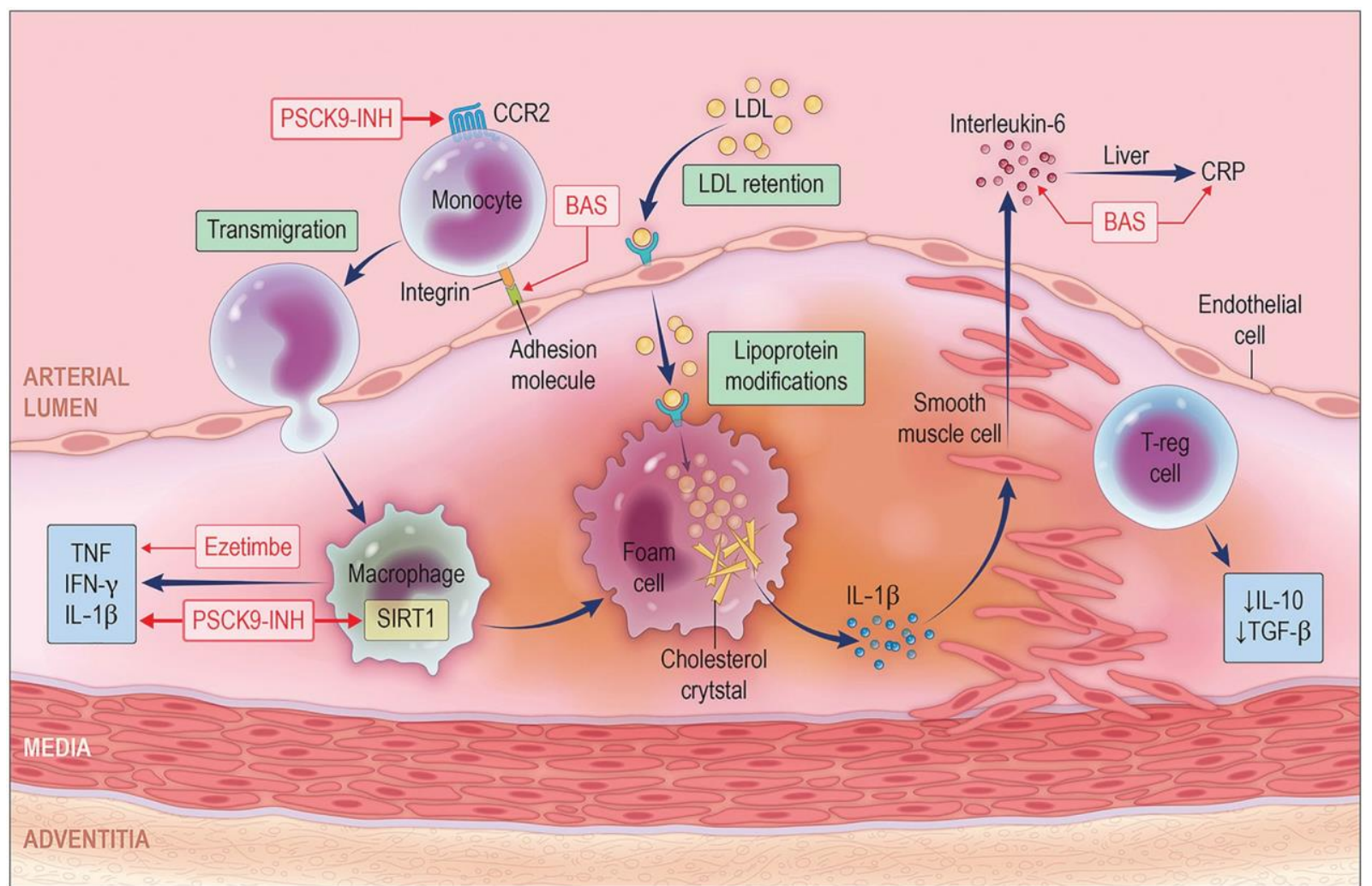

Figure 2. Suggested effects of non-statin lipid lowering drugs on inflammation. The atherosclerotic plaque is similar to the one in Figure 1, here possibly effects on inflammatory pathways are depicted. CCR2: C-C chemokine receptor type 2; LDL: Low-density lipoprotein; CRP: C-reactive protein; TNF: Tumor necrosis factor; IFN: Interferon; IL: Interleukine; SIRT: Sirtuin; TGF: Transforming Growth Factor.

epigenetic modification of relevant genes. Notably, PCSK9 seems to an important regulator of sirtuins, and accordingly, it is possibly that some of the anti-inflammatory effects of PCSK9 inhibition could be mediated through modulation of sirtuins.

Taken together, PCSK9 inhibition does not seem to reduce CRP, but have some promising effects that could modulate the inflammatory arm of atherosclerosis such as inhibiting monocyte influx into the atherosclerotic lesion by downregulating CCR2 expression and modulating the sirtuins in an anti-inflammatory direction. In addition, PCSK9 inhibition has been suggested to attenuate oxidative stress, enhance autophagy and modulate vascular smooth muscle cells to a less pro-atherogenic phenotype. Altogether, these effects that not necessarily will be mirrored by CRP, will have to be proven in larger clinical studies that also include advanced molecular analyses on clinical samples.

\section{Bile acid sequestrants}

Devaraj et al. demonstrated that colsevelam in addition to lowering LDL-cholesterol in mildly hypercholesterolemic subjects also significantly lowered CRP, and there was no correlation between CRP and LDL-cholesterol lowering [48]. McGettingan et al. used a murine model of liver steatosis demonstrating that sevelamer reduced the hepatic cell expression of pro-inflammatory genes including IL-1b, TNF and IL-6 [49]. Fuchs et al. have shown that in a murine model colsevelam reduced inflammation including reduction of the prototypical pro-atherogenic chemokines monocyte chemoattractant protein 1 (MCP-1)/CCL2 and vascular cell adhesion molecule 1 [50]. In a study utilizing a human monocyte line, Mobraten et al. showed that bile acids activate the G-protein coupled receptor TGR5 in parallel with stimulating toll-like receptor 4, leading to an increase in inflammatory cytokines IL-6 and IL-8 [51], possibly indicating that BAS could affect this signaling. Altogether, although data are scarce, these studies may suggest a possible antiinflammatory effect of BAS, potentially at least partly independent of LDL lowering.

\section{Cholesterol absorption inhibitors}

When ezetimibe is added to simvastatin after an acute coronary syndrome more patients get significant reductions in LDL-cholesterol and CRP, corresponding to a reduction in clinical endpoints [52]. Krysiak et al. showed that both ezetimibe and simvastatin reduced TNF and IFNC in people with elevated LDL-cholesterol, however only significant for simvastatin. The reduction in intracellular adhesion molecule 1 and CRP was strongest for the combination of simvastatin and ezetimibe [53]. Qin et al. used a macrophage model to demonstrate that ezetimibe downregulated the TNF gene and further reduced levels of TNF through nuclear factor kappa-light-chain-enhancer of activated B cells/mitogen-activated protein kinase pathway [54]. These in vitro data may suggest that the potential anti-inflammatory effects of ezetimibe are merely secondary to a reduction in LDL-cholesterol. Ghanim et al. observed that when obese persons were fed cream, several inflammatory markers 
increased including IL-1b, TNF and matrix metallopepdidase 9. These markers were significantly reduced by the combination of simvastatin and ezetimibe [55], but this study did not examine the effects of statins and ezetimibe separately. Altogether, although there are some data suggesting that ezetimibe could have anti-inflammatory effects, the effects are most probably less than the effects of statins, and whether the anti-inflammatory effects of ezetimibe are at least partly independent of its effects on LDL-cholesterol is still unclear.

\section{General considerations}

Based on several epidemiological, clinical and experimental studies it seems to be of major importance to target both the lipid and the inflammatory arms of atherogenesis. In addition to therapeutic options that directly target inflammation, some lipid lowering medication may possess anti-inflammatory effects beyond that of LDL-cholesterol lowering that in itself will attenuate inflammation. Such effects are well known for statins. However, whereas current data do not support strong anti-inflammatory effects of BAS and ezetimibe, the anti-inflammatory effects of PSCK9 inhibition should be an area of future interest. In particular, based on the presumably lack of effects on CRP levels, PCSK9 inhibition may modulate other parts of the inflammatory response than statins, and the anti-inflammatory potential of the combination of these drugs should be further explored (Figure 2).

\section{Anti-inflammatory properties - a resource unused?}

As inflammation is a key player both in the initiation and the propagation of the atherosclerotic process, and as the CANTOS trial has shown that anti-inflammatory therapy can have an impact on clinical endpoints - how can we take advantage of the anti-inflammatory properties of drugs already in use and how should we include anti-inflammatory effects in the development of new drugs?

The positive results from the CANTOS trial should be explored deeper in relation to whether non-statin lipid lowering drugs have effects on IL-1b signaling and other antiinflammatory pathways, especially as statin drugs may have different effects on IL-1b signaling in different cells [56]. This is important as the European Lipid guidelines recently advocated a reduction of LDL-cholesterol to below $1.4 \mathrm{mmol} / \mathrm{L}$ in patients with atherosclerotic diseases [36] necessitating non-statin drugs in addition to statins in order to obtain this treatment goal.

\section{Acknowledgements}

We thank Scott Leighton, CMI, Medicus Media for creating the illustrations.

\section{Disclosure statement}

No potential conflict of interest was reported by the author(s).

\section{References}

[1] Libby P, Buring JE, Badimon L, et al. Atherosclerosis. Nat Rev Dis Primers. 2019;5(1):56.

[2] Hovland A, Jonasson L, Garred P, et al. The complement system and toll-like receptors as integrated players in the pathophysiology of atherosclerosis. Atherosclerosis. 2015;241(2): 480494.

[3] Gisterå A, Hansson GK. The immunology of atherosclerosis. Nat Rev Nephrol. 2017;13(6):368-380.

[4] Ekdahl KN, Teramura Y, Hamad OA, et al. Dangerous liaisons: complement, coagulation, and kallikrein/kinin cross-talk act as a linchpin in the events leading to thromboinflammation. Immunol Rev. 2016;274(1):245-269.

[5] Jackson SP, Darbousset R, Schoenwaelder SM. Thromboinflammation: challenges of therapeutically targeting coagulation and other host defense mechanisms. Blood. 2019; 133(9):906-918.

[6] Ridker PM. From C-reactive protein to interleukin-6 to interleukin-1: moving upstream to identify novel targets for atheroprotection. Circ Res. 2016;118(1):145-156.

[7] Stiekema LCA, Stroes ESG, Verweij SL, et al. Persistent arterial wall inflammation in patients with elevated lipoprotein(a) despite strong low-density lipoprotein cholesterol reduction by proprotein convertase subtilisin/kexin type 9 antibody treatment. Eur Heart J. 2019;40(33):2775-2781.

[8] Ridker PM, Everett BM, Thuren T, et al. Antiinflammatory therapy with canakinumab for atherosclerotic disease. $\mathrm{N}$ Engl J Med. 2017;377(12):1119-1131.

[9] Libby P, Interleukin 1. Interleukin-1 beta as a target for atherosclerosis therapy: biological basis of CANTOS and beyond. J Am Coll Cardiol. 2017;70(18): 22782289.

[10] Ridker PM, Everett BM, Pradhan A, et al. Low-dose methotrexate for the prevention of atherosclerotic events. N Engl J Med. 2019;380(8):752-762.

[11] Forero-Pe a DA, Gutierrez FR. Statins as modulators of regulatory T-cell biology. Mediators Inflamm. 2013;2013:167086.

[12] Tousoulis D, Psarros C, Demosthenous M, et al. Innate and adaptive inflammation as a therapeutic target in vascular disease: the emerging role of statins. J Am Coll Cardiol. 2014; 63(23):2491-2502.

[13] Sodha NR, Sellke FW. The effect of statins on perioperative inflammation in cardiac and thoracic surgery. J Thorac Cardiovasc Surg. 2015;149(6):1495-1501.

[14] Li GM, Zhao J, Li B, et al. The anti-inflammatory effects of statins on patients with rheumatoid arthritis: a systemic review and meta-analysis of 15 randomized controlled trials. Autoimmun Rev. 2018;17(3):215-225.

[15] Virchow R. Cellular patology. London: John Churchill; 1858.

[16] Libby P. Inflammation in atherosclerosis. Arterioscler Thromb Vasc Biol. 2012;32(9):2045-2051.

[17] Hansson GK, Hermansson A. The immune system in atherosclerosis. Nat Immunol. 2011;12(3):204-212.

[18] Steinberg D. Atherogenesis in perspective: hypercholesterolemia and inflammation as partners in crime. Nat Med. 2002;8(11): 1211-1217.

[19] M€ nzel T, Camici GG, Maack C, et al. Impact of oxidative stress on the heart and vasculature: part 2 of a 3-part series. J Am Coll Cardiol. 2017;70(2):212-229.

[20] Tabas I, Williams KJ, Boren J. Subendothelial lipoprotein retention as the initiating process in atherosclerosis: update and therapeutic implications. Circulation. 2007;116(16):1832-1844. 
[21] Nahrendorf M, Swirski FK. Cholesterol, CCR2, and monocyte phenotypes in atherosclerosis. Eur Heart J. 2017;38(20): 15941596.

[22] Kuznetsova T, Prange KHM, Glass CK, et al. Transcriptional and epigenetic regulation of macrophages in atherosclerosis. Nat Rev Cardiol. 2020;17(4):216-228.

[23] Samstad EO, Niyonzima N, Nymo S, et al. Cholesterol crystals induce complement-dependent inflammasome activation and cytokine release. J Immunol. 2014;192(6):2837-2845.

[24] Bullenkamp J, Dinkla S, Kaski JC, et al. Targeting T cells to treat atherosclerosis: odyssey from bench to bedside. Eur Heart J Cardiovasc Pharmacother. 2016;2(3):194-199.

[25] Sage AP, Tsiantoulas D, Binder CJ, et al. The role of B cells in atherosclerosis. Nat Rev Cardiol. 2019;16(3):180-196.

[26] Kristiansen TA, Vanhee S, Yuan J. The influence of developmental timing on B cell diversity. Curr Opin Immunol. 2018; 51:7-13.

[27] G'mez-Moreno D, Adrover JM, Hidalgo A. Neutrophils as effectors of vascular inflammation. Eur J Clin Invest. 2018;48: e12940.

[28] Moschonas IC, Tselepis AD. The pathway of neutrophil extracellular traps towards atherosclerosis and thrombosis. Atherosclerosis. 2019;288:9-16.

[29] Jaiswal S, Libby P. Clonal haematopoiesis: connecting ageing and inflammation in cardiovascular disease. Nat Rev Cardiol 2020;17(3):137-144.

[30] Fuster JJ, Walsh K, Mutations S, et al. Somatic mutations and clonal hematopoiesis: unexpected potential new drivers of agerelated cardiovascular disease. Circ Res. 2018;122(3):523-532.

[31] Dorsheimer L, Assmus B, Rasper T, et al. Association of mutations contributing to clonal hematopoiesis with prognosis in chronic ischemic heart failure. JAMA Cardiol. 2019;4(1):25-33.

[32] Robinson JG, Farnier M, Krempf M, et al. Efficacy and safety of alirocumab in reducing lipids and cardiovascular events. $\mathrm{N}$ Engl J Med. 2015;372(16):1489-1499.

[33] Sabatine MS, Giugliano RP, Wiviott SD, et al. Efficacy and safety of evolocumab in reducing lipids and cardiovascular events. N Engl J Med. 2015;372(16):1500-1509.

[34] Sabatine MS, Giugliano RP, Keech AC, et al. Evolocumab and clinical outcomes in patients with cardiovascular disease. N Engl J Med. 2017;376(18):1713-1722.

[35] Schwartz GG, Steg PG, Szarek M, et al. Alirocumab and cardiovascular outcomes after acute coronary syndrome. N Engl J Med. 2018;379(22):2097-2107.

[36] Mach F, Baigent C, Catapano AL, et al. 2019 ESC/EAS guidelines for the management of dyslipidaemias: lipid modification to reduce cardiovascular risk. Eur Heart J. 2020;41(1):111-188.

[37] Spinelli V, Chavez-Talavera O, Tailleux A, et al. Metabolic effects of bile acid sequestration: impact on cardiovascular risk factors. Curr Opin Endocrinol Diabetes Obes. 2016;23(2): 138144.

[38] The Lipid Research Clinics Coronary Primary Prevention Trial results. II. The relationship of reduction in incidence of coronary heart disease to cholesterol lowering. JAMA. 1984;251(3): 365-374.

[39] Garcia-Calvo M, Lisnock J, Bull HG, et al. The target of ezetimibe is Niemann-Pick C1-Like 1 (NPC1L1). Proc Natl Acad Sci USA. 2005;102(23):8132-8137.

[40] Cannon CP, Blazing MA, Giugliano RP, et al. Ezetimibe added to statin therapy after acute coronary syndromes. N Engl J Med. 2015;372(25):2387-2397.
[41] Ouchi Y, Sasaki J, Arai H, et al. Ezetimibe lipid-lowering trial on prevention of atherosclerotic cardiovascular disease in 75 or older (EWTOPIA 75): a randomized, controlled trial. Circulation. 2019;140(12):992-1003.

[42] Cao Y-X, Li S, Liu H-H, et al. Impact of PCSK9 monoclonal antibodies on circulating hs-CRP levels: a systematic review and meta-analysis of randomised controlled trials. BMJ Open. 2018;8(9):e022348.

[43] Tang Z, Jiang L, Peng J, et al. PCSK9 siRNA suppresses the inflammatory response induced by oxLDL through inhibition of NF-jB activation in THP-1-derived P-1-derived macrophages. Int J Mol Med. 2012;30(4):931-938.

[44] Tang ZH, Peng J, Ren Z, et al. New role of PCSK9 in atherosclerotic inflammation promotion involving the TLR4/NF-jB pathway. Atherosclerosis. 2017;262:113-122.

[45] Bernelot Moens SJ, Neele AE, Kroon J, et al. PCSK9 monoclonal antibodies reverse the pro-inflammatory profile of monocytes in familial hypercholesterolaemia. Eur Heart J. 2017; 38(20):1584-1593.

[46] Grune J, Meyborg H, Bezhaeva T, et al. PCSK9 regulates the chemokine receptor CCR2 on monocytes. Biochem Biophys Res Commun. 2017;485(2):312-318.

[47] D'Onofrio N, Vitiello $M$, Casale R, et al. Sirtuins in vascular diseases: emerging roles and therapeutic potential. Biochim Biophys Acta. 2015;1852(7):1311-1322.

[48] Devaraj S, Autret B, Jialal I. Effects of colesevelam hydrochloride (WelChol) on biomarkers of inflammation in patients with mild hypercholesterolemia. Am J Cardiol. 2006;98(5):641-643.

[49] McGettigan BM, McMahan RH, Luo Y, et al. Sevelamer improves steatohepatitis, inhibits liver and intestinal Farnesoid $\mathrm{X}$ Receptor (FXR), and reverses innate immune dysregulation in a mouse model of non-alcoholic fatty liver disease. J Biol Chem. 2016;291(44):23058-23067.

[50] Fuchs CD, Paumgartner G, Mlitz V, et al. Colesevelam attenuates cholestatic liver and bile duct injury in Mdr2-/- mice by modulating composition, signalling and excretion of faecal bile acids. Gut. 2018;67(9):1683-1691.

[51] Mobraten K, Haugbro T, Karlstrom E, et al. Activation of the bile acid receptor TGR5 enhances LPS-induced inflammatory responses in a human monocytic cell line. J Recept Signal Transduct Res. 2015;35(5):402-409.

[52] Bohula EA, Giugliano RP, Cannon CP, et al. Achievement of dual low-density lipoprotein cholesterol and high-sensitivity Creactive protein targets more frequent with the addition of ezetimibe to simvastatin and associated with better outcomes in IMPROVE-IT. Circulation. 2015;132(13):1224-1233.

[53] Krysiak R, Zmuda W, Okopien B. The effect of ezetimibe, administered alone or in combination with simvastatin, on lymphocyte cytokine release in patients with elevated cholesterol levels. J Intern Med. 2012;271(1):32-42.

[54] Qin L, Yang YB, Yang YX, et al. Anti-inflammatory activity of ezetimibe by regulating NF-jB/MAPK pathway in THP-1 macrophages. Pharmacology. 2014;93(1-2):69-75.

[55] Ghanim H, Green K, Abuaysheh S, et al. Ezetimibe and simvastatin combination inhibits and reverses the pro-inflammatory and pro-atherogenic effects of cream in obese patients. Atherosclerosis. 2017;263:278-286.

[56] Liberale L, Carbone F, Camici GG, et al. IL-1b and statin treatment in patients with myocardial infarction and diabetic cardiomyopathy. J Clin Med. 2019;8(11):1764. 